\title{
HETEROSIS INTERPOBLACIONAL EN AGROMORFOLOGÍA Y CAPSAICINOIDES DE CHILES NATIVOS DE OAXACA
}

\section{INTERPOPULATION HETEROSIS FOR AGROMORPHOLOGY AND CAPSAICINOIDS IN NATIVE PEPPERS FROM OAXACA}

\author{
Raquel Martínez-Martínez ${ }^{1}$, Isaura Méndez-Infante ${ }^{1}$, Heidy M. Castañeda-Aldaz ${ }^{2}$, Araceli M. Vera-Guzmán ${ }^{1}$, \\ José L. Chávez-Servia ${ }^{1 \star}$ y J. Cruz Carrillo-Rodríguez ${ }^{2}$
}

${ }^{1}$ CIIDIR-Unidad Oaxaca, Instituto Politécnico Nacional. Hornos \# 1003. 71230, Santa Cruz Xoxocotlán, Oaxaca, México. ${ }^{2}$ Instituto Tecnológico del Valle de Oaxaca, Ex-hacienda Nazareno. 71230, Santa Cruz Xoxocotlán, Oaxaca, México.

${ }^{*}$ Autor para correspondencia: (jchavezs@ipn.mx)

\section{RESUMEN}

Las poblaciones nativas o autóctonas de chile (Capsicum annuum L.) muestran alta heterogeneidad y divergencias, y pueden constituirse como progenitores para formular estrategias genotécnicas que exploren y exploten la máxima heterosis a partir de esas divergencias genéticas. En este trabajo se evaluó la heterosis en capsaicinoides y características agromorfológicas de híbridos interpoblacionales de chile de diferente origen geográfico. Se evaluaron agromorfológicamente ocho progenitores y nueve híbridos, y a la cosecha de frutos se determinó el contenido de capsaicina (CAP) y dihidrocapsaicina (DH) por cromatografía de gases. Se determinaron diferencias significativas $(P<$ 0.01) entre progenitores e híbridos para días a floración, fructificación y maduración de frutos, altura de planta, número y peso de frutos por planta, y longitud y ancho de frutos. Se determinó heterosis tanto positiva como negativa respecto al progenitor medio y mejor progenitor en variables fenológicas, $y$ heterosis positiva respecto al progenitor medio en número y peso de frutos por planta, pero se asoció con menor longitud de fruto. Las poblaciones del tipo Solterito de Oaxaca (C-14) y Bolita de Chiapas (C-18) presentaron los mayores contenidos de capsaicina $\left(>66 \mu \mathrm{g} \mathrm{mL}^{-1}\right)$ y dihidrocapsaicina $\left(>96 \mu \mathrm{g} \mathrm{mL}^{-1}\right)$, y en el caso del chile de Agua el contenido de CAP y DH fue menor a 44 y $42 \mu \mathrm{g} \mathrm{mL} \mathrm{m}^{-1}$, respectivamente. En los híbridos interpoblacionales el promedio en capsaicinoides fue menor que sus progenitores y no hubo diferencias significativas en la heterosis de híbridos respecto a las poblaciones progenitoras, excepto cuando en la cruza intervino el progenitor C-14, morfotipo de mayor contenido de CAP y DH. Los resultados muestran que es posible aprovechar la heterosis que se genera al cruzar poblaciones nativas divergentes morfológicamente, y diferentes en contenido de capsaicinoides y origen geográfico.

Palabras clave: Capsicum annuum, divergencias fenotípicas, cromatografía, híbridos no convencionales, poblaciones nativas.

\section{SUMMARY}

Native populations of pepper (Capsicum annuum L.) are highly heterogeneous and divergent, and some of them may be used as parents to formulate breeding strategies, that explore and exploit maximum heterosis from their genetic divergences. In this work, interpopulation heterosis on agro-morphological traits and capsaicinoids was evaluated on interpopulation hybrids of native pepper from different geographic origin. Eight progenitors and nine hybrids were evaluated agro-morphologically and by gas chromatography (GC) for determination of capsaicin (CAP) and dihydrocapsaicin (DH) content in fruit. Significant differences $(P<0.01)$ were determined among progenitors and hybrids in days to flowering, number of ripening fruits, plant height, weight and number of fruits per plant, and fruit length and width. Positive and negative heterosis compared to midparent and best progenitor were calculated over all phenological traits, and positive heterosis respect to mid-parent was estimated for number and weight of fruits per plant, but in this case it was associated with lower fruit length. The populations of Solterito type from Oaxaca $(\mathrm{C}$ 14) and Bolita from Chiapas (C-18) had the highest content of CAP $\left(>66 \mu \mathrm{g} \mathrm{mL}^{-1}\right.$ ) and DH $\left(>96 \mu \mathrm{g} \mathrm{mL}^{-1}\right)$; in contrast, the chile de Agua populations had the lowest CAP and DH content, with 44 and 42 $\mu \mathrm{g} \mathrm{mL}^{-1}$, respectively. In the interpopulation hybrids, the average in capsaicinoid content was less than in their progenitors; consequently, there were no significant differences in heterosis compared to their parents; except when C-14 was a progenitor, a morphotype with the highest content of CAP and DH. Results indicate that is possible to take advantage of the heterosis generated from crossing of divergent populations in fruit morphology and different content of capsaicinoids and geographic origin.

Index words: Capsicum annuum, phenotypic divergences, gas chromatography, non-conventional hybrids, native populations.

\section{INTRODUCCIÓN}

En México se distribuye una gran variedad de acervos genéticos silvestres y cultivados de chile (Capsicum annuum L. y C. frutescens L.), que se distinguen por una gran variabilidad de formas, tamaños y coloraciones de frutos, picor y características fisiológicas y morfológicas de planta, hojas y flores (Aguilar et al., 2010). Estos acervos han sido utilizados desde la época precolombina (Perry y Flannery, 2007; Long, 2010; Powis et al., 2013). Además, esa diversidad de chiles continúa su evolución en condición silvestre o mediante domesticación, y por ello en diversas regiones se distinguen tipos morfológicos de frutos particulares de relevancia para los consumidores y agricultores locales (Loaiza-Figueroa et al., 1989; Hernández-Verdugo et al., 2001; Latournerie et al., 2002; Votava et al., 2005; CastañónNájera et al., 2008; Alonso et al., 2008; Morán-Bañuelos et al., 2008; Kraft et al., 2010; Moreno-Pérez et al., 2011; Castellón-Martínez et al., 2012). 
Los chiles silvestres y variedades autóctonas tienen alta preferencia regional, y se preservan gracias a la demanda de sus consumidores, y en el caso de las variantes cultivadas es gracias al cuidado de los agricultores. En Oaxaca, aunque existe alta preferencia por los chiles de amplia distribución nacional como Serrano, Jalapeño, de Árbol, Piquín y Pasilla, los chiles regionales que más se consumen son: de Agua, Piquín, Tusta, Paradito y Tabiche, y las razones de consumo obedecen a sabor, aroma, picor y múltiples formas de preparación (Castellón-Martínez et al., 2012). No obstante, tanto en el Estado de Oaxaca como en Yucatán, Tabasco, Chiapas, Veracruz, Puebla y Guerrero, entre otros, existe escaso número de variedades registradas y generadas de las variantes o morfotipos nativos y populares regionalmente. También, el aprovechamiento de la variabilidad local directa, sin un proceso de selección sistemática, hace que en ciertos morfotipos, como el chile de agua de Oaxaca, se presenten serios problemas fitopatológicos durante su cultivo, por siembra de semilla con reducida variabilidad genética o semilla infestada (Vásquez et al., 2009).

La hibridación es una estrategia genotécnica de uso común en el mejoramiento genético de chiles, tanto para mejorar rasgos agronómicos como aspectos de calidad de fruto, vida de anaquel y metabolitos secundarios como capsaicinoides, flavonides y ácido ascórbico. Las variantes más utilizadas son los cruzamientos inter o intraespecíficos (Shifriss y Sacks, 1980; Zewdie y Bosland, 2000; Zewdie y Bosland, 2001; Martínez et al., 2005; Geleta y Labuschagne, 2006; Garcés-Claver et al., 2007; Cruz-Pérez et al., 2007; Payakhapaab et al., 2012; Patil et al., 2012; Hasanuzzaman et al., 2013; Butcher et al., 2013).

En todos los casos se busca explotar la divergencia genética parental para aprovechar la heterosis resultante (Krishnamurthy et al., 2013). De acuerdo con la fuente de germoplasma se generan híbridos convencionales y no convencionales; los primeros se distinguen porque involucran solamente líneas endogámicas con cierto grado de homocigosis, y en el segundo caso se pueden involucrar progenitores no endogámicos o combinaciones de progenitores endogámicos y no endogámicos (Vasal et al., 1994; Espinosa et al., 1999; Espinosa et al., 2000).

En este trabajo se planteó aprovechar la heterosis producida por el cruzamiento población $\times$ población de diferentes orígenes geográficos, con base en los planteamientos de divergencias genéticas y valores heteróticos producidos por cruzamientos interpoblacionales. Por ejemplo, en maíz (Zea mays L.) las divergencias y heterosis fueron aprovechadas por Romero et al. (2002) y Dzib-Aguilar et al. (2011), y en chile Dulce por Pech et al. (2010) para generar materiales sobresalientes. Así, el objetivo de este estudio fue evaluar la heterosis interpoblacional sobre caracteres agromorfológicos y contenido de capsaicinoides de híbridos interpoblacionales de chile nativo con diferente origen geográfico.

\section{MATERIALES Y MÉTODOS}

\section{Material biológico y evaluación agromorfológica en invernadero}

Se utilizaron ocho progenitores de los morfotipos de Capsicum annuum L. denominados regionalmente como chile de Agua, Miahuateco, Paradito o Solterito y Bolita, y nueve híbridos interpoblacionales de cruza simple (población $\times$ población) (Cuadro 1). Aquí se utiliza el término morfotipo para definir una variante morfológica de fruto reconocida por agricultores y consumidores

La semilla de progenitores e híbridos interpoblacionales se germinaron en sustrato comercial Spagnum sp. (Peat moss $($ ) $)$, y se trasplantaron en un invernadero $45 \mathrm{~d}$ después de la siembra, en camas de suelo, estiércol y aserrín desinfectadas con TCMTB:-(tiocianometiltio), benzotiazol (Busan $30 \mathrm{~Wb} \AA$ ) y carbofurán granulado (Furadán 350 $L \circledR)$. El invernadero se encuentra dentro de las instalaciones del Instituto Tecnológico del Valle de Oaxaca, ubicado en la Ex-hacienda de Nazareno Xoxocotlán, Oaxaca, México. Se utilizó riego y fertilizaciones por goteo.

La distribución de tratamientos (progenitores más híbridos) se hizo en un diseño de bloques al azar con tres repeticiones. La fertilización, mediante el sistema de goteo, se hizo inicialmente con la fórmula comercial soluble $15 \mathrm{~N}-30 \mathrm{P}-15 \mathrm{~K}$, después con $13 \mathrm{~N}-6 \mathrm{P}-18 \mathrm{~K}$, y en fructificación con $12 \mathrm{~N}-00 \mathrm{P}-46 \mathrm{~K}$ más nitrato de calcio. Para el control de plagas y enfermedades se utilizó N-triclorometiltio4-ciclohexeno-1,2-dicarboximida (Captan $\AA)$, oxicloruro de cobre (Cupravit mix ${ }^{\circledR}$ ), clorotalonil (Daconil $\AA$ ), sulfato de cobre y extractos vegetales comerciales (Bio-crak®).

La evaluación agromorfológica se hizo en todos los híbridos y progenitores, pero en el análisis de resultados no se incluyeron los datos agromorfológicos del progenitor C-04 ni de las cruzas C-04 × C-03, C-07 × C-14, C-10 × $\mathrm{C}-18$ y C-19 $\times$ C-03 porque presentaron insuficientes plantas sobrevivientes ( $<5$ plantas en las tres repeticiones), a pesar del programa de control químico de enfermedades. No obstante, sí se consideraron en la evaluación de capsaicionoides. Para la descripción agromorfológica se tomó como referencia los descriptores de Capsicum del IPGRI (IPGRI, 1995); por ejemplo, días del trasplante a floración, fructificación y maduración de frutos de la primera y segunda ramificación se registraron cuando más de $50 \%$ de las plantas alcanzaron cada etapa fenológica. 
Cuadro 1. Descripción general de progenitores e híbridos de cruzas simples de chile (C. annuum L.).

\begin{tabular}{|c|c|c|c|c|}
\hline Progenitores & Morfotipo distintivo & Origen & Híbridos & Genealogía \\
\hline C-03 & Miahuateco & San José Miahuatlán, Puebla & $\mathrm{HCH} 3$ & $\mathrm{C}-04 \times \mathrm{C}-03$ \\
\hline C-04 & Chile de Agua & Etla, Oaxaca & $\mathrm{HCH} 6$ & $\mathrm{C}-07 \times \mathrm{C}-03$ \\
\hline C-07 & Chile de Agua & San Juan Guelavia, Oaxaca & $\mathrm{HCH} 9$ & $\mathrm{C}-07 \times \mathrm{C}-14$ \\
\hline C-08 & Chile de Agua & Zimatlán, Oaxaca & HCH17 & $\mathrm{C}-08 \times \mathrm{C}-18$ \\
\hline C-10 & Chile de Agua & Sola de Vega, Oaxaca & $\mathrm{HCH} 19$ & $\mathrm{C}-10 \times \mathrm{C}-03$ \\
\hline C-14 & Paradito o Solterito & Nazareno Xoxocotlán, Oaxaca & $\mathrm{HCH} 21$ & $\mathrm{C}-10 \times \mathrm{C}-18$ \\
\hline C-18 & Bolita & Tuxtla Gtz., Chiapas & $\mathrm{HCH} 24$ & $\mathrm{C}-19 \times \mathrm{C}-03$ \\
\hline \multirow[t]{2}{*}{ C-19 } & Chile de agua & San Sebastián Etla, Oaxaca & $\mathrm{HCH} 27$ & C- $19 \times \mathrm{C}-14$ \\
\hline & & & $\mathrm{HCH} 30$ & $\mathrm{C}-19 \times \mathrm{C}-18$ \\
\hline
\end{tabular}

\section{Determinación de capsaicinoides}

La determinación de capsaicina (CAP) y dihidrocapsaicina $(\mathrm{DH})$ se hizo en muestras de frutos maduros de color rojo o amarillo, según el morfotipo de progenitores e híbridos. Se utilizó el método de cromatografía de gases (CG) propuesto por Abraham-Juárez et al. (2008) y con algunas modificaciones de Vera-Guzmán et al. (2011). Los frutos se secaron en un horno a $50^{\circ} \mathrm{C}$ de 2 a $3 \mathrm{~d}$, hasta alcanzar peso constante. Posteriormente se molieron y transfirieron a frascos ámbar para almacenarse a $4{ }^{\circ} \mathrm{C}$ hasta su análisis. La identificación y cuantificación de CAP y DH se determinó mediante la curva de calibración de dos estándares: capsaicina (8-Methyl-N-vanillyl-trans-6-nonenamide; Reg. 2816484 Sigma) con $95 \%$ de pureza, y dihidrocapsaicina (8-Methyl-N-vanillylnonanamide; Reg. 2815150 Sigma) con $90 \%$ de pureza, ambos sintetizados de Capsicum. Cada muestra se analizó por triplicado y los resultados se reportaron en $\mu \mathrm{g} \mathrm{mL}^{-1}$.

\section{Análisis estadístico}

Con base en el diseño establecido se hicieron análisis de varianza con el objetivo de avaluar las diferencias entre tratamientos (híbridos y progenitores), y posteriormente una comparación de medias por el método de Tukey $(\mathrm{P}<0.05)$ tanto para las variables agromorfológicas como para capsaicinoides.

La heterosis se estimó con base en el progenitor medio y mejor progenitor (Fehr, 1987; Falconer y Mackay, 1996), mediante las expresiones siguientes: porcentaje de heterosis respecto al progenitor medio $H P M=\left[\left(F_{1}-P M\right) / P M\right] \times 100$ y mejor progenitor $H B=\left[\left(F_{1}-B P\right) / B P\right] \times 100$, donde: $P M=$ media de los progenitores $=\left[\left(P_{1}+P_{2}\right) / 2\right]$, y $B P=$ media del mejor progenitor que interviene en la cruza $\left(P_{1}\right.$ o $\left.P_{2}\right)$.

Para evaluar la significancia de la heterosis respecto al progenitor medio y al mejor progenitor se utilizó la prueba de " $t$ " propuesta por Wynne et al. (1970): respecto al progenitor medio $H P M(t): \quad t=\frac{(F 1-P M)}{\sqrt{\left[\frac{3}{2 r}(C M E)\right]}}$

y respecto al mejor progenitor $H B(t): t=\frac{(F 1-B P)}{\left.\sqrt{\left[\frac{3}{2 r}(C M E)\right.}\right]}$,

donde: $F_{1}=$ media del híbrido $\mathrm{F}_{1}$ para el carácter específico, $P M=$ progenitor medio $\left[\left(P_{1}+P_{2}\right) / 2\right], B P=$ media del mejor progenitor $\left(P_{1}\right.$ o $\left.P_{2}\right), C M E=$ cuadrado medio del error, $\mathrm{y} r=$ número de repeticiones.

\section{RESULTADOS Y DISCUSIÓN}

\section{Variación agromorfológica y heterosis interpoblacional}

Hubo diferencias significativas entre tratamiento (progenitores e híbridos) en nueve variables agromorfológicas evaluadas (Cuadro 2). En este ensayo las variables de mayor variación fueron número y peso de frutos por planta, con coeficientes de variación superiores a $30 \%$. Los resultados muestran que los progenitores e híbridos difieren en uno o más características agromorfológicas.

En este trabajo, los días a floración, fructificación y maduración de frutos de las primeras ramificaciones fueron indicadores de precocidad en las poblaciones evaluadas. Así, entre progenitores las poblaciones Paradito de Oaxaca (C-14) y Bolita de Chiapas (C-18) fueron las más tardías en alcanzar las etapas fenológicas, y entre híbridos la cruza C-19 × C-18 fue la más tardía. En general, floración, fructificación y maduración de frutos fueron ligeramente más tardías en las poblaciones híbridas que en los progenitores, pero se determinó el efecto inverso en altura de planta, peso y número de frutos por planta (Cuadro 3). Con la hibridación se busca mayor precocidad en la progenie pero en este caso el efecto fue contrario, aunque se compensó con mayor peso promedio de frutos por planta. Los frutos más largos 
Cuadro 2. Significancia de cuadrados medios, promedios y coeficientes de variación (CV) de nueve variables evaluadas en cinco híbridos y siete progenitores.

\begin{tabular}{lccc}
\hline Variable & Cuadrados medios & Media & CV (\%) \\
\hline Días a floración $^{\dagger}$ & $964^{* *}$ & 41.9 & 17.5 \\
Días a fructificación $^{\dagger}$ & $1483^{* *}$ & 47.2 & 12.4 \\
Días a maduración de frutos $^{\dagger}$ & $3035^{* *}$ & 83.6 & 3.7 \\
Altura a $60 \mathrm{ddt}^{\dagger}(\mathrm{cm})$ & $1149^{* *}$ & 67.8 & 16.2 \\
Altura a $\left.120 \mathrm{ddt}^{* *} \mathrm{~cm}\right)$ & $1515^{* *}$ & 138.8 & 6.1 \\
Número de frutos por planta & $15,153^{* *}$ & 70.9 & 37.0 \\
Peso de frutos por planta $(\mathrm{g})$ & $1,788,580^{* *}$ & 1054.1 & 35.3 \\
Longitud de fruto $(\mathrm{cm})$ & $57^{* *}$ & 7.6 & 11.1 \\
Ancho de fruto $(\mathrm{cm})$ & $5^{* *}$ & 2.7 & 8.3 \\
\hline
\end{tabular}

${ }^{\dagger} \mathrm{ddt}=$ días después del trasplante; ${ }^{*}$ significativo a $\mathrm{P}<0.01$.

$(>10 \mathrm{~cm})$ se encontraron en el progenitor C-08 e híbridos C-07 × C-03 y C-10 × C-03, y en el extremo opuesto están C-14 (Paradito) y C-18 (Bolita) con menos de $6 \mathrm{~cm}$ en longitud y ancho de fruto. En este trabajo, los progenitores de chile de Agua presentaron variaciones en longitud de 9.0 a $10.5 \mathrm{~cm}$ y corresponden con los valores reportados por Martínez-Sánchez et al. (2010) para este mismo morfotipo.

En relación con la heterosis en agromorfología se determinaron diferencias significativas importantes de los híbridos interpoblacionales respecto al progenitor medio y mejor progenitor para todas las variables evaluadas, excepto en ancho de fruto. El primer patrón distintivo fue que los híbridos fueron tardíos a floración, fructificación y maduración, pero hubo excepciones: C- $07 \times \mathrm{C}-03$ y C- $19 \times$ $\mathrm{C}$-14. En número de frutos y peso de frutos por planta los híbridos C- $08 \times$ C-18, C-19 × C-14 y C-19 × C-18 superaron significativamente al comportamiento medio de sus progenitores, pero decreció el tamaño de fruto (Cuadro 4). Esto es, se incrementó el número y peso de frutos pero el tamaño se afectó. Esta respuesta es de singular importancia cuando se quiere mejorar el tamaño medio de frutos, principalmente hacia los morfotipos de chile de Agua. En otros casos se incrementó el número de frutos pero decreció el peso y longitud.

Los resultados de efectos heteróticos interpoblacionales favorables en fenología, altura de planta y características de frutos, indican que los cruzamientos población $\times$ población generan híbridos con mayor o menor vigor que sus progenitores, a pesar de su condición heterogénea o no endogámica, donde las divergencias geográficas de origen de las poblaciones pueden suponerse, hasta cierto punto, como divergencias genéticas (Shifriss y Sacks, 1980; Krishnamurthy et al., 2013). Es decir, el aislamiento geográfico de las poblaciones nativas de chile favorecen las divergencias genéticas, y probablemente es mayor cuando están bajo cultivo y aislados geográficamente. Esta misma hipótesis también se confirma con los trabajos interpoblacionales encontrados en chile Dulce (Pech et al., 2010), y en maíz (Zea mays L.) (Romero et al., 2002).

\section{Capsaicinoides y heterosis}

En los análisis de varianza se determinaron diferencias significativas entre tratamientos en contenido de capsaicinas (CAP) y dihidrocapsaicinas $(\mathrm{DH})$, y en la relación CAP/DH. Los progenitores C-14 (Paradito) y C-18 (Bolita) presentaron el mayor contenido de CAP $\left(>40 \mu \mathrm{g} \mathrm{mL}^{-1}\right)$ y de $\mathrm{DH}\left(>95 \mu \mathrm{g} \mathrm{mL}^{-1}\right)$. Estos patrones de alto contenido de CAP y DH coinciden con Zewdie y Bosland (2001) quienes reportaron dos accesiones de C. pubescens que tenían alto contenido de CAP y DH. En los casos de C-07 (Agua), C-10 (Agua), C-18 (Bolita) y C-19 (Agua) presentaron mayor contenido de dihidrocapsaicinas que de capsaicinas. En el caso de los híbridos interpoblacionales, el contenido de CAP y DH fue menor que en los progenitores; no obstante, se incrementó el contenido de dihidrocapsaicina hasta el punto de mantener un valor semejante al de capsaicina o ser mayor. Es decir, en general se mantuvo una relación 1:1 en CAP:DH (Cuadro 5).

En estudios previos realizados por Vera-Guzmán et al. (2011), se reporta un contenido de capsaicina y dihidrocapsaicina para chile de Agua de 4.9 y $1.6 \mu \mathrm{g} \mathrm{mL}^{-1}$, y para Solterito o Paradito de 142.0 y $65.5 \mu \mathrm{g} \mathrm{mL} \mathrm{m}^{-1}$, respectivamente. Ahora, esos mismos morfotipos presentaron valores de 28.3 a $43.1 \mu \mathrm{g} \mathrm{mL}^{-1}$ de CAP y de 23.0 a $41.3 \mu \mathrm{g} \mathrm{mL}^{-1}$ de DH para chile de Agua, y para Solterito de 208.3 y 156.1 $\mu \mathrm{g} \mathrm{mL} \mathrm{L}^{-1}$ de CAP y DH, respectivamente. Esto confirma que las variantes de chile de Agua presentan menor contenido de CAP y DH, en referencia al mayor contenido de ambos compuestos en Solterito de Oaxaca; es decir, se corrobora el alto picor en Solterito y bajo en chile de Agua. 
Cuadro 3. Valores promedio de características fisiológicas y agromorfológicas evaluadas en progenitores e híbridos del chile (Capsicum annuum L.), en invernadero.

\begin{tabular}{|c|c|c|c|c|c|c|c|c|c|}
\hline $\begin{array}{l}\text { Progenitores e } \\
\text { híbridos }\end{array}$ & $\begin{array}{l}\text { Floración } \\
\left(_{\text {días })^{\dagger}}\right.\end{array}$ & $\begin{array}{l}\text { Fructificación } \\
\text { (días) }\end{array}$ & $\begin{array}{l}\text { Maduración } \\
\text { (días) }\end{array}$ & $\begin{array}{c}\text { Altura a } 60 \\
\mathrm{ddt}^{\dagger}(\mathrm{cm})\end{array}$ & $\begin{array}{l}\text { Altura a } 120 \\
\text { ddt }(\mathrm{cm})\end{array}$ & $\begin{array}{l}\text { Número de } \\
\text { frutos por } \\
\text { planta }\end{array}$ & $\begin{array}{l}\text { Peso de frutos } \\
\text { por planta }(\mathrm{g})\end{array}$ & $\begin{array}{l}\text { Longitud } \\
\text { fruto }(\mathrm{cm})\end{array}$ & $\begin{array}{l}\text { Ancho de } \\
\text { fruto }(\mathrm{cm})\end{array}$ \\
\hline \multicolumn{10}{|c|}{ Progenitores } \\
\hline C-03 & $37.3 \mathrm{bcd}^{\ddagger}$ & $40.2 \mathrm{cde}$ & $83.7 \mathrm{c}$ & $83.0 \mathrm{ab}$ & $169.2 \mathrm{a}$ & $61.0 \mathrm{~b}$ & $1490.5 \mathrm{ab}$ & $8.6 \mathrm{~b}$ & $3.0 \mathrm{c}$ \\
\hline C-07 & $26.5 \mathrm{~d}$ & $30.3 \mathrm{e}$ & $57.8 \mathrm{f}$ & $65.8 \mathrm{abc}$ & $120.0 \mathrm{e}$ & $46.8 \mathrm{~b}$ & $1247.7 \mathrm{abc}$ & $9.1 \mathrm{ab}$ & $3.1 \mathrm{bc}$ \\
\hline C-14 & $63.0 \mathrm{a}$ & $66.0 \mathrm{~b}$ & $119.5 \mathrm{ab}$ & $65.7 \mathrm{abc}$ & $155.8 \mathrm{ab}$ & $31.3 \mathrm{~b}$ & $162.3 \mathrm{~d}$ & $5.9 c$ & $1.4 \mathrm{e}$ \\
\hline C-18 & $58.3 \mathrm{a}$ & $82.0 \mathrm{a}$ & $121.7 \mathrm{a}$ & $45.2 \mathrm{c}$ & $138.0 \mathrm{~cd}$ & $62.7 \mathrm{~b}$ & $41.7 \mathrm{~d}$ & $1.2 \mathrm{e}$ & $1.1 \mathrm{e}$ \\
\hline C-19 & $32.3 \mathrm{bcd}$ & 36.3 cde & $64.0 \mathrm{de}$ & $75.2 \mathrm{ab}$ & $125.0 \mathrm{de}$ & $37.0 \mathrm{~b}$ & $1228.8 \mathrm{abc}$ & $9.9 \mathrm{ab}$ & $3.5 \mathrm{abc}$ \\
\hline Promedio & 40.3 & 46.8 & 82.2 & 64.6 & 135.7 & 44.2 & 940.4 & 7.7 & 2.7 \\
\hline $\mathrm{C}-08 \times \mathrm{C}-18$ & $43.8 \mathrm{~b}$ & $47.8 \mathrm{c}$ & $85.0 \mathrm{c}$ & $64.8 \mathrm{abc}$ & $136.7 \mathrm{~cd}$ & $206.0 \mathrm{a}$ & $910.8 \mathrm{bc}$ & $4.1 \mathrm{~d}$ & $2.1 \mathrm{~d}$ \\
\hline $\mathrm{C}-10 \times \mathrm{C}-03$ & $41.8 \mathrm{bc}$ & $45.8 c$ & $87.2 \mathrm{c}$ & 83.7 a & $142.7 \mathrm{bc}$ & $55.2 \mathrm{~b}$ & $1595.7 \mathrm{ab}$ & $10.4 \mathrm{a}$ & $3.6 \mathrm{ab}$ \\
\hline $\mathrm{C}-19 \times \mathrm{C}-14$ & $39.7 \mathrm{bc}$ & $42.7 \mathrm{~cd}$ & $83.3 c$ & $80.7 \mathrm{a}$ & $157.5 \mathrm{ab}$ & $100.0 \mathrm{~b}$ & $1065.0 \mathrm{bc}$ & $8.4 \mathrm{~b}$ & $2.3 \mathrm{~d}$ \\
\hline $\mathrm{C}-19 \times \mathrm{C}-18$ & $62.0 \mathrm{a}$ & $65.0 \mathrm{~b}$ & $108.3 \mathrm{~b}$ & $48.3 \mathrm{c}$ & $142.0 \mathrm{bc}$ & $118.7 \mathrm{ab}$ & $696.5 \mathrm{~cd}$ & $4.0 \mathrm{~d}$ & $2.2 \mathrm{~d}$ \\
\hline Promedio & 44.2 & 47.8 & 85.6 & 72.4 & 143.1 & 108.2 & 1213.3 & 7.5 & 2.7 \\
\hline
\end{tabular}

†ddt, días después del trasplante; ${ }^{*}$ Promedios de una columna con la misma letra no son diferentes estadísticamente (Tukey, 0.05).

En relación a la respuesta heterótica respecto al progenitor medio (PM) y mejor progenitor (BP), se observó, en general, que no hubo diferencias significativas respecto a los progenitores en CAP, DH y relación CAP/DH, excepto la heterosis negativa y significativa en referencia al mejor progenitor en el híbrido C-07 $\times$ C-14 (Agua $\times$ Paradito) tanto para CAP como DH, y en C-19 $\times$ C-14 (Agua $\times$ Paradito) solo en $\mathrm{DH}$ (Cuadro 6). Esto es, al cruzar poblaciones de menor contenido de capsaicinoides (p. ej., chile de Agua $\times$ chile de Agua), la composición no cambia significativamente respecto a los progenitores, a diferencia de la respuesta que obtuvo Garcés-Claver et al. (2007) al cruzar 'Yolo Wonder' $\times$ Serrano Criollo de Morelos, donde el híbrido formado fue superior a los progenitores.

En los casos en que el progenitor materno fue chile de Agua y el progenitor paterno presentó alto picor (p. ej., Solterito): C-08 × C-18, C-10 × C-18 y C-19 × C-18, el nivel de capsaicina y dihidrocapsaicina en la progenie no superó al progenitor medio ni al mejor progenitor (Cuadro 6). Esta respuesta se atribuye a un probable efecto materno, como determinó Sánchez-Sánchez et al. (2010) en cruzamientos poblacionales de C. pubescens.

Otro de los cruzamientos de interés agronómico eran las combinaciones o hibridaciones interpoblacionales entre chile de Agua de Oaxaca $\times$ Miahuateco de Puebla (pericarpio verde claro $\times$ pericarpio verde oscuro). En principio, en los híbridos evaluados $(\mathrm{C}-04 \times \mathrm{C}-03, \mathrm{C}-07 \times \mathrm{C}-03, \mathrm{C}-10 \times$ C-03 y C-19 $\times$ C-03) dominó el pericarpio oscuro, se incrementó el peso y tamaño de frutos por planta, y predominó el mayor contenido de dihidrocapsaicina en relación al de capsaicina. Sin embargo, en heterosis tanto del progenitor medio como del mejor progenitor, no hubo diferencias significativas respecto a los progenitores en capsaicinoides. Es decir, se pueden mejorar las variantes de chile de Agua de Oaxaca al utilizar como fuente al chile Miahuateco de Puebla, sin alterar las características morfológicas de fruto ni el contenido de capsaicinoides, excepto la coloración oscura de pericarpio (Cuadros 5 y 6 ).

\section{CONCLUSIONES}

Los progenitores e híbridos interpoblacionales evaluados de chile presentaron diferencias significativas $(\mathrm{P}<0.01)$ en características agromorfológicas, contenidos de capsaicina (CAP) y dihidrocapsaicina (DH), y en la relación CAP/DH. Es decir, tanto las poblaciones progenitoras e híbridos mostraron divergencias fenotípicas y heterogeneidad poblacional. Se detectaron heterosis positivas y negativas significativas en las progenies respecto al progenitor medio y mejor progenitor, en rasgos fenológicos, número y peso de frutos 
Cuadro 4. Porcentajes de heterosis de cinco híbridos y sus diferencias estadísticas respecto al progenitor medio (PM) y mejor progenitor (BP).

\begin{tabular}{|c|c|c|c|c|}
\hline $\begin{array}{l}\text { Híbrido } \\
(\stackrel{x}{x} \text { ㅇ })\end{array}$ & Heterosis & Floración $\left(\right.$ días) ${ }^{\dagger}$ & Fructificación (días) ${ }^{\dagger}$ & Maduración (días) $\dagger$ \\
\hline \multirow{2}{*}{$\mathrm{C}-07 \times \mathrm{C}-03$} & $\mathrm{PM}$ & $5.0^{* *}$ & $6.4^{* *}$ & $-9.1^{* *}$ \\
\hline & $\mathrm{BP}$ & $-10.3^{* *}$ & $-6.6^{* *}$ & $-23.1^{\star *}$ \\
\hline \multirow{2}{*}{$\mathrm{C}-08 \times \mathrm{C}-18$} & $\mathrm{PM}$ & $1.5^{\star \star}$ & $-16.2^{* *}$ & $-6.2^{* *}$ \\
\hline & $\mathrm{BP}$ & $-24.9^{* *}$ & $-41.7^{\star *}$ & $-30.1^{\star *}$ \\
\hline \multirow{2}{*}{$\mathrm{C}-10 \times \mathrm{C}-03$} & $\mathrm{PM}$ & $13.0^{* *}$ & $13.4^{* *}$ & $14.1^{* *}$ \\
\hline & $\mathrm{BP}$ & $12.0^{* *}$ & $12.7^{\star *}$ & $4.2^{* *}$ \\
\hline \multirow{2}{*}{$\mathrm{C}-19 \times \mathrm{C}-14$} & PM & $-16.8^{\star *}$ & $-16.6^{* *}$ & $-9.2^{\star *}$ \\
\hline & $\mathrm{BP}$ & $-37.0^{* *}$ & $-35.3^{* *}$ & $-30.3^{\star *}$ \\
\hline \multirow{2}{*}{ C- $19 \times C-18$} & $\mathrm{PM}$ & $36.8^{\star *}$ & $9.9^{* *}$ & $16.7^{\star *}$ \\
\hline & $\mathrm{BP}$ & $6.3^{* *}$ & $-20.7^{\star *}$ & $-11.0^{* *}$ \\
\hline $\begin{array}{l}\text { Híbrido } \\
\left(\hat{\partial} \mathrm{x}+{ }^{\prime}\right)\end{array}$ & $\begin{array}{l}\text { Número de frutos por } \\
\text { planta }\end{array}$ & $\begin{array}{l}\text { Peso de fruto por } \\
\text { planta }(\mathrm{g})\end{array}$ & Longitud de fruto $(\mathrm{cm})$ & Ancho de fruto $(\mathrm{cm})$ \\
\hline \multirow{2}{*}{$\mathrm{C}-07 \times \mathrm{C}-03$} & $13.1^{\star \star}$ & $31.4 \mathrm{~ns}$ & $19.2^{\star *}$ & $14.9 \mathrm{~ns}$ \\
\hline & $0.0 \mathrm{~ns}$ & $20.7^{\star \star}$ & $15.8^{* *}$ & $12.7 \mathrm{~ns}$ \\
\hline \multirow{2}{*}{ C- $08 \times C-18$} & $294.9^{* *}$ & $19.2^{* *}$ & $-29.3^{* *}$ & $-13.9 \mathrm{~ns}$ \\
\hline & $228.7^{* *}$ & $-38.7^{* *}$ & $-60.6^{* *}$ & $-44.8 \mathrm{~ns}$ \\
\hline \multirow{2}{*}{$\mathrm{C}-10 \times \mathrm{C}-03$} & $22.4^{\star *}$ & $32.1^{* *}$ & $18.8^{\star *}$ & $13.4 \mathrm{~ns}$ \\
\hline & $-9.6^{* *}$ & $7.1^{* *}$ & $15.8^{* *}$ & $8.4 \mathrm{~ns}$ \\
\hline \multirow{2}{*}{ C- $19 \times C-14$} & $192.7^{\star *}$ & $53.1^{* *}$ & $6.5 \mathrm{~ns}$ & $-7.4 \mathrm{~ns}$ \\
\hline & $170.3^{* *}$ & $-13.3^{* *}$ & $-14.8^{\star *}$ & $-35.1 \mathrm{~ns}$ \\
\hline \multirow{2}{*}{$\mathrm{C}-19 \times \mathrm{C}-18$} & $138.1^{\star *}$ & $9.6^{* *}$ & $-27.9^{* *}$ & $-3.5 \mathrm{~ns}$ \\
\hline & $89.4^{\star *}$ & $-43.3^{* \star}$ & $-59.6^{\star *}$ & $-36.8 \mathrm{~ns}$ \\
\hline
\end{tabular}

${ }^{\dagger} \mathrm{ddt}$, días después del trasplante; $\mathrm{ns}=$ diferencias no significativas a $\mathrm{P}>0.05$; ${ }^{* *}$ diferencia significativa a $\mathrm{P}<0.01$.

por planta, y longitud de fruto, lo que indica que fueron superiores o inferiores al valor promedio de los progenitores, respectivamente. Respecto al contenido de capsaicinas y dihidrocapsaicinas, en general el porcentaje de heterosis de la progenie respecto a los progenitores no fue significativo, excepto en los cruzamientos donde intervino el morfotipo Solterito o Paradito. En los cruzamientos de poblaciones de contenido bajo de capsaicinoides se generaron híbridos interpoblacionales también con bajo contenido de capsacinoides. Esta segregación es relevante para productores y consumidores que prefieren chiles de Agua no picosos o de bajo picor.

\section{BIBLIOGRAFÍA}

Abraham-Juárez M. R., M. C. Rocha-Granados, M. G. López, R. F. Rivera-Bustamante and M. Ochoa-Alejo (2008) Virus-induced silencing of Comt, pAmt and Kas genes results in a reduction of capsaicinoids accumulation in chili pepper fruits. Planta 227:681-695
Aguilar V. H., T. Corona, P. López, L. Latournerie, M. Ramírez, H. Villalón y J. A. Aguilar (2010) Los Chiles de México y su Distribución. SINAREFI, Colegio de Posgraduados, INIFAP, ITConkal, UANL y UAN. Texcoco, México. 114 p.

Alonso R. A., C. Moya, A. Cabrera, P. Ponce, R. Quiroga, M. A. Rosales y J. L. Zuart (2008) Evaluación in situ de la variabilidad genética de los chiles silvestres (Capsicum spp.) en la región Frailesca del estado de Chiapas, México. Cultivos Tropicales 29:49-55.

Butcher J. D., K. M. Crosby, K. S. Yoo, B. Patil, J. L. Jifon and W. L. Rooney (2013) Heterosis in different $\mathrm{F}_{1}$ Capsicum annuum genotypes for fruit traits, ascorbic acid, capsaicin and flavonoids. Scientia Horticulture 159:72-79.

Castañón-Nájera G., L. Latournerie-Moreno, M. Mendoza-Elos, A. Vargas-López y H. Cárdenas-Morales (2008) Colección y caracterización de chile (Capsicum spp.) en Tabasco, México. Revista Internacional de Botánica Experimental 77:189-202.

Castellón-Martínez E., J. L. Chávez-Servia, J. C. Carrillo-Rodríguez y A. M. Vera-Guzmán (2012) Preferencia de consumo de chiles (Capsicum annuum L.) nativos en los Valles Centrales de Oaxaca, México. Revista Fitotecnia Mexicana 35(Núm. Esp. 5):27-35.

Cruz-Pérez A. B., V. A. González-Hernández, R. M. Soto-Hernández, M. A. Gutiérrez-Espinosa, A. A. Gardea-Béjar y M. PérezGrajales (2007) Capsaicinoides, vitamina $\mathrm{C} y$ heterosis durante el desarrollo del fruto de chile manzano. Agrociencia 41:627-635.

Dzib-Aguilar L. A., J. C. Segura-Correa, R. Ortega-Paczka y L. Latournerie-Moreno (2011) Cruzas dialélica entre poblaciones nativas 
Cuadro 5. Significancia de cuadrados medios y promedios del contenido de capsaicinoides en progenitores e híbridos cruza simple de chiles nativos de Oaxaca.

\begin{tabular}{|c|c|c|c|}
\hline Progenitores e híbridos & $\begin{array}{c}\text { Capsaicina } \\
\left(\mathrm{CAP}, \mu \mathrm{g} \mathrm{mL} \mathrm{mL}^{-1}\right)\end{array}$ & $\begin{array}{l}\text { Dihidrocapsaicina } \\
\left(\mathrm{DH}, \mu \mathrm{g} \mathrm{mL} \mathrm{mL}^{-1}\right)\end{array}$ & $\begin{array}{c}\text { Relación CAP/ } \\
\text { DH }\end{array}$ \\
\hline \multicolumn{4}{|c|}{ Cuadrados medios } \\
\hline Tratamientos & $5816.6^{\star *}$ & $3331.1^{\star *}$ & $0.462^{* *}$ \\
\hline \multicolumn{4}{|c|}{ Progenitores } \\
\hline C-03 & $15.9 \mathrm{fg}^{\dagger}$ & $12.0 \mathrm{~h}$ & $1.33 \mathrm{bc}$ \\
\hline C-04 & 30.9 def & $20.3 \mathrm{gh}$ & $1.52 \mathrm{~b}$ \\
\hline C-07 & 28.6 defg & 41.3 cde & 0.69 ef \\
\hline C-08 & $43.1 \mathrm{~cd}$ & $23.0 \mathrm{fgh}$ & $1.88 \mathrm{a}$ \\
\hline C-10 & $16.0 \mathrm{fg}$ & 34.4 defg & $0.47 \mathrm{gh}$ \\
\hline C-14 & $208.3 \mathrm{a}$ & $156.1 \mathrm{a}$ & $1.34 \mathrm{bc}$ \\
\hline C-18 & $66.5 \mathrm{~b}$ & $96.3 \mathrm{~b}$ & 0.69 ef \\
\hline C-19 & 28.3 defg & 41.0 cde & $0.69 \mathrm{ef}$ \\
\hline Promedio & 54.7 & 53.0 & 1.08 \\
\hline \multicolumn{4}{|c|}{ Híbridos interpoblacionales } \\
\hline C-04 × C-03 & $24.1 \mathrm{efg}$ & 36.9 cdefg & $0.65 \mathrm{fg}$ \\
\hline $\mathrm{C}-07 \times \mathrm{C}-03$ & 28.0 defg & $38.8 \mathrm{cdef}$ & $0.72 \mathrm{ef}$ \\
\hline $\mathrm{C}-07 \times \mathrm{C}-14$ & $50.4 \mathrm{bc}$ & $50.4 \mathrm{~cd}$ & $0.99 \mathrm{~d}$ \\
\hline $\mathrm{C}-08 \times \mathrm{C}-18$ & $27.8 \mathrm{defg}$ & 40.5 cde & $0.68 \mathrm{ef}$ \\
\hline $\mathrm{C}-10 \times \mathrm{C}-03$ & $17.2 \mathrm{fg}$ & $13.5 \mathrm{~h}$ & $1.28 \mathrm{c}$ \\
\hline $\mathrm{C}-10 \times \mathrm{C}-18$ & 41.7 cde & $47.4 \mathrm{~cd}$ & $0.88 \mathrm{de}$ \\
\hline $\mathrm{C}-19 \times \mathrm{C}-03$ & $11.1 \mathrm{~g}$ & $30.8 \mathrm{efg}$ & $0.36 \mathrm{~h}$ \\
\hline $\mathrm{C}-19 \times \mathrm{C}-14$ & $53.0 \mathrm{bc}$ & $53.3 \mathrm{c}$ & $0.99 \mathrm{~d}$ \\
\hline C-19 × C-18 & $52.6 \mathrm{bc}$ & $52.9 \mathrm{c}$ & $0.99 \mathrm{~d}$ \\
\hline Promedio & 34.0 & 40.5 & 0.84 \\
\hline
\end{tabular}

${ }^{*}$ diferencia significativa a $\mathrm{P}<0.01$; ${ }^{\dagger}$ En columna, medias con la misma letra no difieren significativamente (Tukey, 0.05).

de maíz de Yucatán y poblaciones mejoradas. Tropical and Subtropical Agoecosystems 14:119-127.

Espinosa A., M. Tadeo y A. Tapia (1999) Variedades mejoradas no convencionales de maíz para agrosistemas de mediana productividad. Agricultura Técnica en México 25:83-87.

Espinosa A., M. Tadeo y A. Tapia-Naranjo (2000) Variedades no convencionales como opción para elevar la productividad de maíces locales en Valles Altos de México. Agronomía Mesoamericana 11:159-161.

Fehr W. R. (1987) Principles of Cultivar Development. Vol. I. Theory and Technique. MacMillan Publishing Company. New York, USA. $536 \mathrm{p}$.

Falconer D. S. and T. F. C. Mackay (1996) Introduction to Quantitative Genetics. Logman Group Ltd, Harlow, England. 464 p.

Garcés-Claver A., R. Gil-Ortega, A. Álvarez-Fernández and M. S. Arnedo-Andrés (2007) Inheritance of capsaicin and dihydrocapsaicin, determined by HPLC-ESI/MS, in a intraspecific cross of Capsicum annuum L. Journal of Agricultural and Food Chemistry 55:6951-6957.

Geleta L. F. and M. T. Labuschagne (2006) Estimate of combining ability for agronomic traits in pepper (Capsicum annuum L.). South African Journal of Plant and Soil 23:73-77.

Hasanuzzaman M., M. A. Hakim, M. M. Hanafi, A. Shukor-Juraimi, M. M. Islam and A. K. M. Shamsuddin (2013) Study of heterosis in Bangladeshi chilli (Capsicum annuum L.) landraces. Agro- ciencia 47:683-690.

Hernández-Verdugo S., R. Luna-Reyes and K. Oyama (2001) Genetic structure and differentiation of wild and domesticated populations of Capsicum annuum (Solanaceae) from Mexico. Plant Systematics and Evolution 226:129-142.

IPGR, International Plant Genetic Resources (1995) Descriptores para Capsicum spp. Instituto Internacional de Recursos Fitogenéticos, Centro Asiático para el Desarrollo e Investigación Relativo a los Vegetales y Centro Agronómico Tropical de Investigación. Roma, Italia. 52 p.

Kraft K. H., J. J. Luna-Ruiz and P. Gepts (2010) Different seed selection and conservation practices for fresh market and dried chile farmers in Aguascalientes, Mexico. Economic Botany 64: 319328.

Krishnamurthy S. L., A. M. Rao, K. M. Reddy, S. Ramesh, S. Hittalmani and M. G. Rao (2013) Limits of parental divergence for the occurrence of heterosis through morphological and AFLP marker in chilli (Capsicum annuum L.). Current Science 104:738-746.

Latournerie L., J. L. Chávez, M. Pérez, G. Castañón, S. A. Rodríguez, L. M. Arias and P. Ramírez (2002) Valoración in situ de la diversidad morfológica de chiles (Capsicum annuum L. y Capsicum chinense Jacq.) en Yaxcabá, Yucatán. Revista Fitotecnia Mexicana 25:25-33.

Loaiza-Figueroa F., K. Rotland, J. A. Laborde and S. D. Tanksley (1989) Patterns of genetic variation of the genus Capsicum (Solanaceae) 
Cuadro 6. Porcentaje de heterosis de nueve híbridos y sus diferencias estadísticas con respecto al progenitor medio (PM) y mejor progenitor (BP), en capsaicina (CAP), dihidrocapsaicina (DH) y relación CAP/DH.

\begin{tabular}{|c|c|c|c|c|c|c|}
\hline $\begin{array}{l}\text { Progenitor } \\
\text { materno }\end{array}$ & $\begin{array}{l}\text { Progenitor } \\
\text { paterno }\end{array}$ & Híbridos cruza simple & Heterosis & CAP & DH & $\mathrm{CAP} / \mathrm{DH}$ \\
\hline \multirow{2}{*}{ C-04 } & \multirow{2}{*}{$\mathrm{C}-03$} & \multirow{2}{*}{$\begin{array}{l}\text { Agua } \times \text { Miahuateco } \\
\quad(\mathrm{C}-04 \times \mathrm{C}-03)\end{array}$} & $\mathrm{PM}$ & $3.0 \mathrm{~ns}$ & $128.5 \mathrm{~ns}$ & $-54.4 \mathrm{~ns}$ \\
\hline & & & $\mathrm{BP}$ & $-22.0 \mathrm{~ns}$ & $81.8 \mathrm{~ns}$ & $-57.2^{\star *}$ \\
\hline \multirow{2}{*}{ C-07 } & \multirow{2}{*}{ C-03 } & \multirow{2}{*}{$\begin{array}{l}\text { Agua } \times \text { Miahuateco } \\
\quad(\mathrm{C}-07 \times \mathrm{C}-03)\end{array}$} & $\mathrm{PM}$ & $25.8 \mathrm{~ns}$ & $45.6 \mathrm{~ns}$ & $-28.7 \mathrm{~ns}$ \\
\hline & & & $\mathrm{BP}$ & $-2.1 \mathrm{~ns}$ & $-6.1 \mathrm{~ns}$ & $-45.9 \mathrm{~ns}$ \\
\hline \multirow{2}{*}{$\mathrm{C}-07$} & \multirow{2}{*}{ C-14 } & \multirow{2}{*}{$\begin{array}{c}\text { Agua } \times \text { Paradito } \\
(\mathrm{C}-07 \times \mathrm{C}-14)\end{array}$} & $\mathrm{PM}$ & $-57.5 \mathrm{~ns}$ & $-48.9 \mathrm{~ns}$ & $-2.5 \mathrm{~ns}$ \\
\hline & & & $\mathrm{BP}$ & $-75.8^{*}$ & $-67.7^{\star *}$ & $-26.1 \mathrm{~ns}$ \\
\hline \multirow{2}{*}{ C-08 } & \multirow{2}{*}{ C- 18} & \multirow{2}{*}{$\begin{array}{l}\text { Agua } \times \text { Bolita amarillo } \\
\quad(\mathrm{C}-08 \times \mathrm{C}-18)\end{array}$} & $\mathrm{PM}$ & $-49.3 n s$ & $-32.1 \mathrm{~ns}$ & $-47.1 \mathrm{~ns}$ \\
\hline & & & $\mathrm{BP}$ & $-58.2 \mathrm{~ns}$ & $-57.9 \mathrm{~ns}$ & $-63.8^{\star *}$ \\
\hline \multirow{2}{*}{$\mathrm{C}-10$} & \multirow{2}{*}{ C-03 } & \multirow{2}{*}{$\begin{array}{l}\text { Agua } \times \text { Miahuateco } \\
\quad(\mathrm{C}-10 \times \mathrm{C}-03)\end{array}$} & $\mathrm{PM}$ & $7.8 \mathrm{~ns}$ & $-41.8 \mathrm{~ns}$ & $42.2 \mathrm{~ns}$ \\
\hline & & & $\mathrm{BP}$ & $7.5 \mathrm{~ns}$ & $-60.8 \mathrm{~ns}$ & $-3.8 \mathrm{~ns}$ \\
\hline \multirow{2}{*}{$\mathrm{C}-10$} & \multirow{2}{*}{ C-18 } & \multirow{2}{*}{$\begin{array}{l}\text { Agua } \times \text { Bolita amarillo } \\
\quad(\mathrm{C}-10 \times \mathrm{C}-18)\end{array}$} & $\mathrm{PM}$ & $1.1 \mathrm{~ns}$ & $-27.5 \mathrm{~ns}$ & $51.7 \mathrm{~ns}$ \\
\hline & & & $\mathrm{BP}$ & $-37.3 \mathrm{~ns}$ & $-50.8 \mathrm{~ns}$ & $27.5 \mathrm{~ns}$ \\
\hline \multirow{2}{*}{ C-19 } & \multirow{2}{*}{ C-03 } & \multirow{2}{*}{$\begin{array}{c}\text { Agua } \times \text { Miahuateco } \\
(\mathrm{C}-19 \times \mathrm{C}-03)\end{array}$} & $\mathrm{PM}$ & $-49.8 \mathrm{~ns}$ & $16.2 \mathrm{~ns}$ & $-64.4 \mathrm{~ns}$ \\
\hline & & & $\mathrm{BP}$ & $-60.8 \mathrm{~ns}$ & $-24.9 \mathrm{~ns}$ & $-72.9 \mathrm{~ns}$ \\
\hline \multirow{2}{*}{ C-19 } & \multirow{2}{*}{ C- 14} & \multirow{2}{*}{$\begin{array}{c}\text { Agua } \times \text { Paradito } \\
(\mathrm{C}-19 \times \mathrm{C}-14)\end{array}$} & $\mathrm{PM}$ & $-55.2 \mathrm{~ns}$ & $-45.9 \mathrm{~ns}$ & $-2.5 \mathrm{~ns}$ \\
\hline & & & $\mathrm{BP}$ & $-74.6 \mathrm{~ns}$ & $-65.9^{* *}$ & $-26.1 \mathrm{~ns}$ \\
\hline \multirow{2}{*}{ C-19 } & \multirow{2}{*}{ C-18 } & \multirow{2}{*}{$\begin{array}{c}\text { Agua } \times \text { Bolita amarillo } \\
(\mathrm{C}-19 \times \mathrm{C}-18)\end{array}$} & $\mathrm{PM}$ & $11.0 \mathrm{~ns}$ & $-22.9 \mathrm{~ns}$ & $43.5 \mathrm{~ns}$ \\
\hline & & & $\mathrm{BP}$ & $-20.9 \mathrm{~ns}$ & $-45.1 \mathrm{~ns}$ & $43.5 \mathrm{~ns}$ \\
\hline
\end{tabular}

ns $=$ diferencia no significativa $(\mathrm{P}>0.05)$; ${ }^{\star}$ diferencia significativa a $\mathrm{P}<0.05 ;{ }^{* *}$ diferencia significativa a $\mathrm{P}<0.01$.

in Mexico. Plant Systematics and Evolution 165:159-188.

Long J. (2010) Los senderos prehispánicos del Capsicum. In: Caminos y Mercados de México. J. Long y A. Attolini (Coords.) UNAM e INAH Serie Histórica General 23. México, D.F. pp:79-105.

Martínez G., J. R. A. Dorantes, M. Ramírez, A. de la Rosa y O. Pozo (2005) Efectos genéticos y heterosis en la vida de anaquel del chile serrano. Revista Fitotecnia Mexicana 28:327-332.

Morán-Bañuelos S. H., V. H. Aguilar-Rincón, T. Corona-Torres, F. Castillo-González, R. M. Soto-Hernández y R. San MiguelChávez (2008) Capsaicinoides en chiles nativos de Puebla, México. Agrociencia 42:807-816.

Moreno-Pérez E. C., C. H. Avendaño-Arrazate, R. Mora-Aguilar, J. Cadena-Iñiguez, V. H. Aguilar-Rincón y J. F. Aguirre-Medina (2011) Diversidad morfológica en colectas de chile guajillo (Capsicum annuum L.) del Centro-Norte de México. Revista Chapingo Serie Horticultura 17:23-30.

Martínez-Sánchez D., M. Pérez-Grajales, J. E. Rodríguez-Pérez y E. C. Moreno-Pérez (2010) Colecta y caracterización morfológica de 'chile de agua' (Capsicum annuum L.) en Oaxaca, México. Revista Chapingo Serie Horticultura 16:169-176.

Payakhapaab S., D. Boonyakiat and M. Nikornpun (2012) Evaluation of heterosis and combining ability of yield components in chillies. Journal of Agricultural Science 4:154-161.

Patil B. T., M. N. Bhalekar and K. G. Shinde (2012) Heterosis studies in chilli (Capsicum annuum L.) for earliness, growth and green fruit yield. Vegetable Science 39:73-75.

Pech A. M., G. Castañón, J. M. Tun, M. Mendoza, J. O. Mijangos, A. Pérez y L. Latournerie (2010) Efectos heteróticos y aptitud combinatoria en poblaciones de chile dulce (Capsicum annuum L.). Revista Fitotecnia Mexicana 33:353-360.

Perry L. and K. V. Flannery (2007) Precolumbian use of chili peppers in the Valley of Oaxaca, Mexico. Proceedings of the National Academy of Science of the USA 104:11905-11909.

Powis T. G., E. Gallaga, R. Lesure, R. Lopez, L. Grivetti, H. Kucera and N. W. Gaikwad (2013) Prehispanic use of chili peppers in Chiapas, Mexico. PLos One 8:e79013. doi:10.1371/journal. pone.0079013.

Romero J., F. Castillo y R. Ortega (2002) Cruzas de poblaciones nativas de maíz de la raza Chalqueño: II. Grupos genéticos, divergencia genética y heterosis. Revista Fitotecnia Mexicana 25:107115.

Sánchez-Sánchez H., V. A. González-Hernández, A. B. Cruz-Pérez, M. Pérez-Grajales, M. A. Gutiérrez-Espinosa, A. A. GardeaBéjar y M. A. Gómez-Lim (2010) Herencia de capsaicinoides en chile manzano (Capsicum pubescens R. \& P.). Agrociencia 44:655-665.

Shifriss C. and J. M. Sacks (1980) The effect of distance between parents on the yield of sweet pepper $\times$ hot pepper hybrids, Capsicum annuum L. in a single harvest. Theoretical and Applied Genetics 58:253-256.

Vasal S. K., N. Vergara and S. McLean (1994) Comportamiento de híbridos de maíz intersintético generados de selección recíproca recurrente. Agronomía Mesoamericana 5:131-134.

Vásquez A., B. Tlapal, M. J. Yáñez, R. Pérez y M. Quintos (2009) Etiología de la marchitez del 'chile de agua' (Capsicum annuum L.) en Oaxaca, México. Revista Fitotecnia Mexicana 32:127-134.

Vera-Guzmán A. M., J. L. Chávez-Servia, J. C. Carrillo-Rodríguez and M. G. López (2011) Phytochemical evaluation of wild and cultivated pepper (Capsicum annuum L. and C. pubescens Ruiz \& Pav.) from Oaxaca, Mexico. Chilean Journal of Agricultural Research 71:578-585.

Votava E. J., J. B. Baral and P. W. Bosland (2005) Genetic diversity of chile (Capsicum annuum var. annuum L.) landraces from Northern Mexico, Colorado and Mexico. Economic Botany 59:8-17.

Zewdie Y. and P. W. Bosland (2000) Capsaicinoid inheritance in an interspecific hybridization of Capsicum annuum $\times$ C. chinense. Journal of the American Society for Horticultural Science 36:13151317. 
Zewdie Y. and P. W. Bosland (2001) Combining ability and heterosis for capsaicinoids in Capsicum pubescens. HortScience 36:13151317.
Wynne J. C., D. A. Emery and P. H. Rice (1970) Combining ability estimation in Arachis hypogaea L. II. Field performance of $\mathrm{F}_{1}$ hybrids. Crop Science 10:713-715. 